\title{
ON A PROBLEM OF COMMUTATIVITY OF AUTOMORPHISMS
}

\author{
M. ANWAR CHAUDHRY and A.B. THAHEEM \\ Department of Mathematical Sciences \\ King Fahd University of Petroleum and Minerals \\ Dhahran 31261, SAUDI ARABIA
}

(Received January 8, 1996)

\begin{abstract}
In this note we provide a partial answer to a problem proposed by $M$. Brešar. We prove that if $\alpha, \beta$ are automorphisms of a commutative prime ring of characteristic not equal to 2 satisfying the equation $\alpha+\alpha^{-1}=\beta+\beta^{-1}$, then either $\alpha=\beta$ or $\alpha=\beta^{-1}$. As a consequence $\alpha$ and $\beta$ commute and in this situation the equation itself ensures the commutativity of $\alpha$ and $\beta$.
\end{abstract}

KEY WORDS AND PHRASES: Prime ring, automorphism.

1991 AMS SUBJECT CLASSIFICATION CODES: Primary 16W20; Secondary 46L40.

The equation

$$
\alpha+\alpha^{-1}=\beta+\beta^{-1}
$$

where $\alpha$ and $\beta$ are automorphisms of a von Neumann algebra has been extensively studied This equation (in case $\alpha$ and $\beta$ commute) has played an important role in the study of Tomita-Takesaki theory $[1,2,3]$. Several conditions have been considered where the equation $\alpha+\alpha^{-1}=\beta+\beta^{-1}$ itself implies the commutativity of $\alpha$ and $\beta$ and thus making the additional assumption that $\alpha$ and $\beta$ commute as redundant. For instance, it has been shown in [4] that if $M$ is a commutative semisimple Banach algebra and $\alpha, \beta$ are automorphisms of $M$ satisfying equation (*), then an application of Gelfand's theory implies that $\alpha$ and $\beta$ commute. Also, it has been shown in [5] that if $\alpha$ and $\beta$ are $*$-automorphisms of a $C^{*}$-algebra $A$ satisfying equation (*) and if either $\alpha$ or $\beta$ is inner, then $\alpha$ and $\beta$ commute. Recently Brešar $[6,7]$ has studied this equation on prime and semiprime rings and has remarkably extended most of the decomposition results of $[4,8]$ on von Neumann algebras about this equation to semiprime and prime rings, using purely algebraic techniques. As an application of Posner's result for $(\alpha, \beta)$-derivations, Brešar [6, Corollary 3] has shown the following generalization of a result of Thaheem $[4,8]$.

THEOREM A. Let $R$ be a prime ring of characteristic not 2. Suppose that automorphisms $\alpha, \beta$ of $R$ satisfy $\alpha+\alpha^{-1}=\beta+\beta^{-1}$. If $\alpha$ and $\beta$ commute then either $\alpha=\beta$ or $\alpha=\beta^{-1}$.

In [6] Brešar has proposed an open question whether or not the assumption that $\alpha$ and $\beta$ commute can be removed in Theorem $A$. In this note we are precisely concerned with this question and provide a partial answer to his problem. We prove that in case $R$ is commutative then the assumption of commutativity of $\alpha$ and $\beta$ can indeed be removed from Theorem A. We prove the following theorem:

THEOREM B. Let $R$ be a commutative prime ring of characteristic not 2. Suppose that automorphisms $\alpha, \beta$ of $R$ satisfy $\alpha+\alpha^{-1}=\beta+\beta^{-1}$. Then either $\alpha=\beta$ or $\alpha=\beta^{-1}$. 
PROOF. It follows from the equation

$$
\alpha+\alpha^{-1}=\beta+\beta^{-1}
$$

that for any $x \in R$,

$$
(\alpha-\beta)\left(x^{2}\right)=\left(\beta^{-1}-\alpha^{-1}\right)\left(x^{2}\right) .
$$

Rewriting (2), we obtain $\alpha\left(x^{2}\right)-\beta\left(x^{2}\right)=\beta^{-1}\left(x^{2}\right)-\alpha^{-1}\left(x^{2}\right)$. That is,

$$
(\alpha(x))^{2}-(\beta(x))^{2}=\left(\beta^{-1}(x)\right)^{2}-\left(\alpha^{-1}(x)\right)^{2} .
$$

Since $R$ is commutative, then using (1) we may rewrite (3) as

$$
(\alpha(x)-\beta(x))(\alpha(x)+\beta(x))=(\alpha(x)-\beta(x))\left(\beta^{-1}(x)+\alpha^{-1}(x)\right)
$$

or what is same

$$
(\alpha(x)-\beta(x))\left(\alpha(x)+\beta(x)-\beta^{-1}(x)-\alpha^{-1}(x)\right)=0 .
$$

By equation (1), we may rewrite (4) as

$$
(\alpha(x)-\beta(x))\left(\beta(x)+\beta^{-1}(x)-\alpha^{-1}(x)+\beta(x)-\beta^{-1}(x)-\alpha^{-1}(x)\right)
$$

or equivalently

$$
2(\alpha(x)-\beta(x))\left(\beta(x)-\alpha^{-1}(x)\right)=0 .
$$

In view of the commutativity of $R$, equation (6) implies that for any $y \in R, 2(\alpha(x)-\beta(x)) y(\beta(x)-$ $\left.\alpha^{-1}(x)\right)=0$. Since $R$ is prime and characteristic of $R$ is not 2 , therefore we have $\alpha(x)-\beta(x)=0$ or $\beta(x)-\alpha^{-1}(x)=0$ for any $x \in R$. Thus either $\alpha=\beta$ or $\alpha=\beta^{-1}$. This completes the proof.

It follows from the conclusion of the above theorem that $\alpha$ and $\beta$ commute. In other words, the equation $\alpha+\alpha^{-1}=\beta+\beta^{-1}$ ensures the commutativity of $\alpha$ and $\beta$. It would be interesting to resolve the problem for certain types of noncommutative prime rings

ACKNOWLEDGMENT. The authors gratefully acknowledge the support provided by King Fahd University of Petroleum and Minerals during this research.

\section{REFERENCES}

[1] HAAGERUP, U. and HANCHE-OLSEN, H., Tomita-Takesaki theory for Jordan algebras, $J$. Operator Theory, 11 (1984), 343-364.

[2] HAAGERUP, U. and SKAU, C.K., Geometric aspects of the Tomita-Takesaki theory - II, Math. Scand, 48 (1981), 241-252.

[3] VAN DAELE, A., A new approach to the Tomita-Takesaki theory of generalized Hilbert algebras, J. Funct. Anal., 15 (1974), 387-393.

[4] THAHEEM, A.B., On a decomposition of a von Neumann algebra, Rend. Sem. Mat. Univ. Padova, 65 (1981), 1-7.

[5] THAHEEM, A.B., On a functional equation on $C^{*}$-algebras, Funkcial, Ekvac., 31 (1988), 411 413.

[6] BREŚAR, M., On the composition of $(\alpha, \beta)$-derivations of rings and applications to von Neumann algebras, Acta Scl. Math., 56 (1992), 369-375.

[7] BREŠAR, M., On certain pairs of automorphisms of rings, J. Austral. Math. Soc. (Series A), 54 (1993), 29-38.

[8] THAHEEM, A.B. and AWAMI, M., A short proof of a decomposition theorem of a von Neumann algebra, Proc. Amer. Math. Soc., 92 (1984), 81-82. 


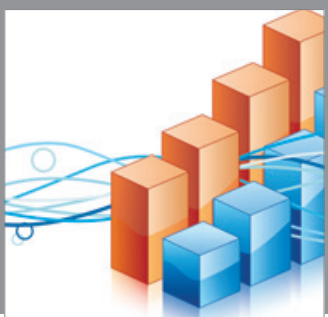

Advances in

Operations Research

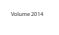

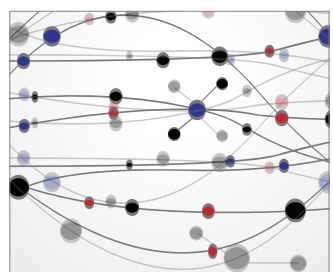

\section{The Scientific} World Journal
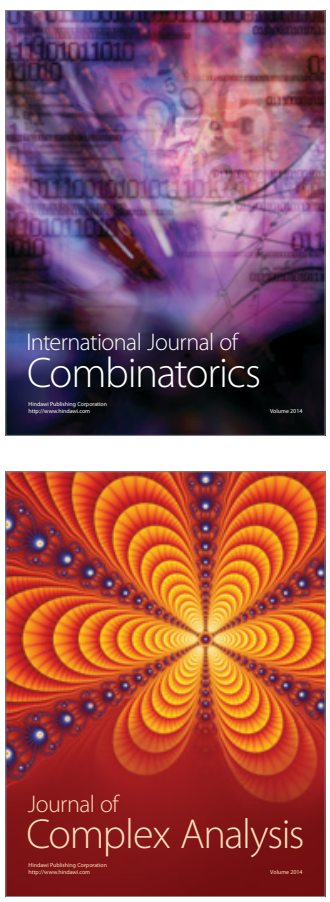

International Journal of

Mathematics and

Mathematical

Sciences
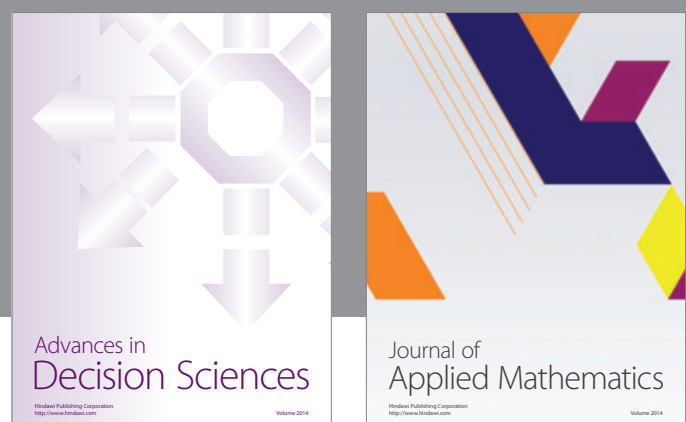

Journal of

Applied Mathematics
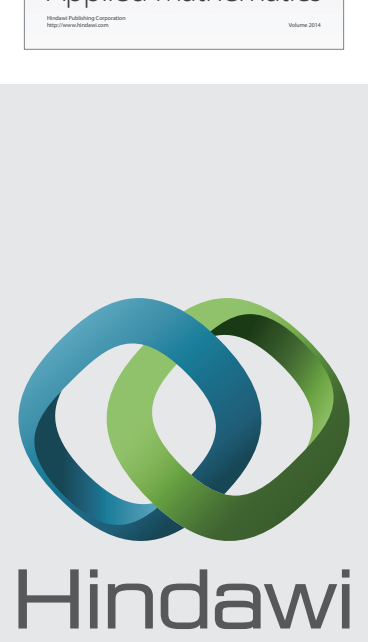

Submit your manuscripts at http://www.hindawi.com
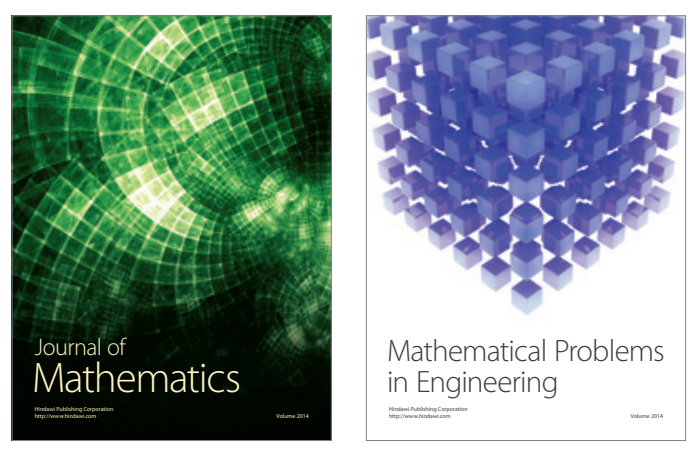

Mathematical Problems in Engineering
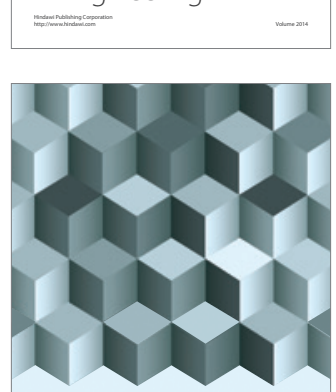

Journal of

Function Spaces
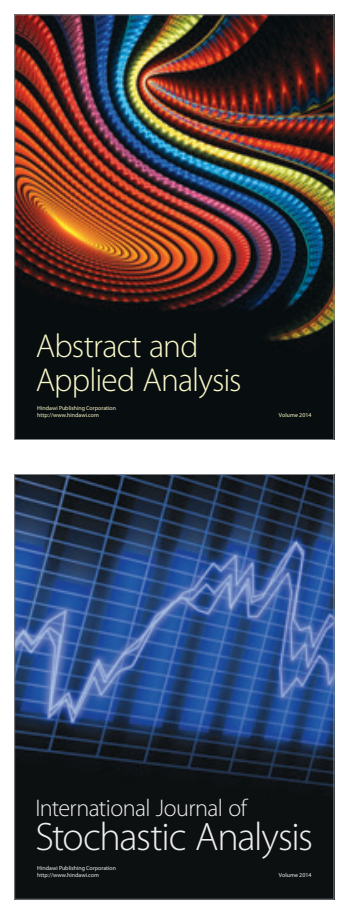

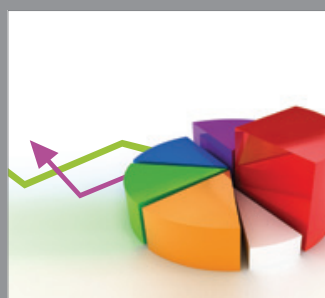

ournal of

Probability and Statistics

Promensencen
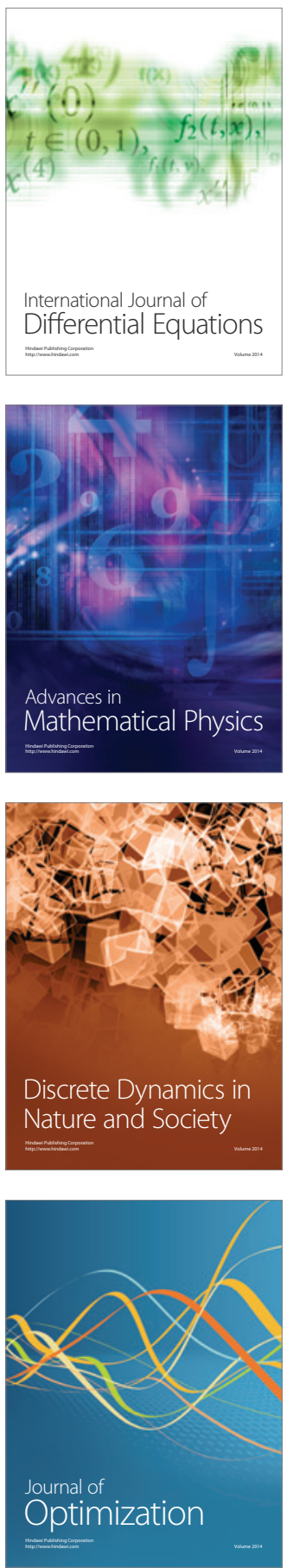